\title{
UV-Visible Spectrophotometric Method and Validation of Organic Compounds
}

\author{
Abdul Moiz Mohammed
}

\begin{abstract}
Absorption and emission of radiation by a compound at different wavelengths in visible and ultra violet region forms an analytical tool for identification of organic compounds. Researchers and scientists from different fields of chemistry and life sciences are constantly in search of an alternate ways to analyze the chemical reactions involving organic and inorganic compounds. The UV-Visible spectroscopic technique serves as an important and simple technique in finding concentration of molecules in a solution, identification the functional groups identification of conjugated organic compounds. The molar absorption coefficient is an intrinsic property of species, and may be treated as a fingerprint to an organic compound. This papers presents a systematic and detailed validation of organic compounds with the molar absorption coefficient in UV-visible region.
\end{abstract}

Index Terms-Electromagnetic; Spectrum; Ultraviolet; Visible; Transition; Molar Absorption Coefficient; Organic; Inorganic.

\section{INTRODUCTION}

A very small portion of electromagnetic spectrum i.e., wavelength $380 \mathrm{~nm}$ to $780 \mathrm{~nm}$ is sensitive to human eye, and is termed as visible spectrum; whereas, ultraviolet region: far ultraviolet $(10 \mathrm{~nm}$ to $200 \mathrm{~nm})$ and near ultraviolet (200 $\mathrm{nm}$ to $380 \mathrm{~nm}$ ). The term spectroscopy is derived from two Latin work "spectron" meaning spirit and "skopien" meaning looking into the world. Spectroscopy in the ultraviolet and visible regions of the spectrum is referred to as electronic spectroscopy, as it involves excitations of electronic states [1]

The electromagnetic radiation is considered to possess both wave nature as well as particles known as photons [2], [3]. Electrons in an atom or molecule are excited, if the frequency of the incident radiation corresponds to the difference in energy state of two electronic states. The electronic structure of the molecules defines the energy difference; thus the proton of lower energy is sufficient for vibrational changes. A plot between absorption and the wavelength $(\mathrm{nm})$ of incident radiation is termed as absorption spectrum. For, UV-Vis region, the absorption spectrum of a molecule depicts a very sharp lines; where each line corresponds to a wavelength equals to the energy needed to excite for the electronic transition. However, the UV-Visible spectrum of most molecules are found to have humps, which is due to the electronic transition accompanied by vibrational levels of higher electronic states. Furthermore, each of the vibrational levels are

Published on March 13, 2018

A. M. Mohammed is with the Department of Basic Sciences, University of Hail, P.O. Box \# 2440, Hail, Saudi Arabia. (e-mail: mr.mamoiz@gmail.com). associated with an even smaller rotational change, they influence each other and blur the sharp spectral lines into bands or broad spectrum [3].

\section{THEORETICAL PRINCIPLES}

\section{A. Quantitative Analysis}

Beer - Lambert laws is widely used in UV-visible spectroscopy, for quantitative analysis such as concentration determination; and qualitative analysis such as identification of molecule properties. The Beer - Lambert law is given as:

$$
A=\varepsilon l c
$$

where, A is the absorbance $\left(=\log \left(\frac{\mathrm{I}_{0}}{\mathrm{I}_{1}}\right)\right)$, I is the light intensity, $\varepsilon$ is molar absorption coefficient, 1 is the sample length and $\mathrm{c}$ is the concentration.

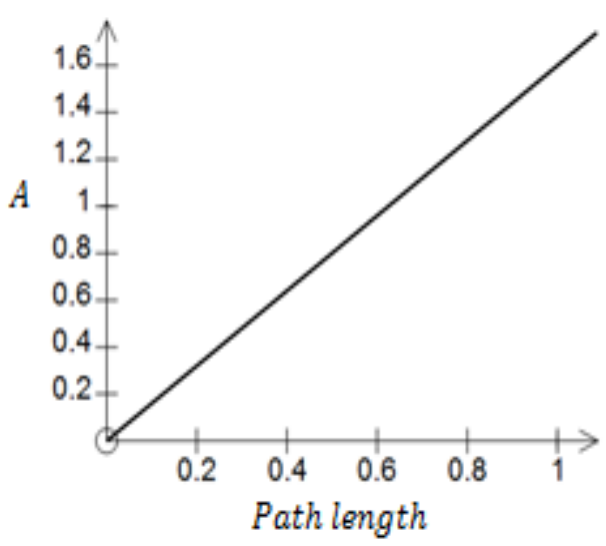

Fig. 1(a). Absorbance $(A)$ versus path length

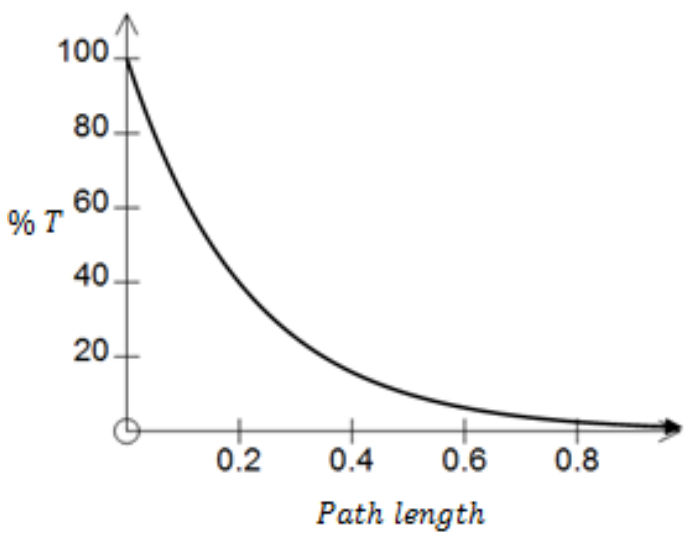

Fig. 1(b). Trasmittance (T) versus path length

The molar absorptivity is a constant for a particular wavelength, Table III shows the molar absorptivity of 
different compounds. If a spectrum is expressed as absorbance (A) as a function of wavelength $(\lambda)$, the derivative spectra are:

$$
\begin{array}{cll}
\text { Zero Order: } & A=f(\lambda) & \Rightarrow A=\varepsilon l c \\
\text { First Order: } & \frac{d A}{d \lambda}=f^{\prime}(\lambda) & \Rightarrow \frac{d A}{d \lambda}=\frac{d \varepsilon}{d \lambda} l c \\
\text { Second Order: } & \frac{d^{2} A}{d \lambda^{2}}=f^{\prime \prime}(\lambda) & \Rightarrow \frac{d^{2} A}{d \lambda^{2}}=\frac{d^{2} \varepsilon}{d \lambda^{2}} l c
\end{array}
$$

The effects of derivatization and graphs for the derivatization of absorbance band is mentioned in detail elsewhere [4]. In order to quantify a single component, wavelength selection is difficult, as the absorbance spectra consists of peaks in both directions. Identification of peak at maximum wavelength $\left(\lambda_{\max }\right)$ is easier for odd-order derivative which crosses the zero point, while peaks appears to be maximum \& minimum for even order derivative. The difference of the maximum and minimum at $\lambda_{\max }$ results in low noises in the absorption spectrum.

With the advent of modern instrument, the analysis of multi-component has become more precise, compared to classical instruments. The principle involved in multicomponent analysis is, sum of the absorbance of each component at any wavelength of a mixture equates the absorbance at that wavelength [5].

$$
A_{\text {total }}=A_{1}+A_{2}+A_{3} \cdots
$$

The numbers of constituent in a mixture is directly related to the number of wavelengths employed to determine the absorbance. The idea is to select wavelengths at which individual component has maximum absorption. Using Beer Lambert law, the values of molar absorptivity of two substances namely, $\mathrm{P}$ and $\mathrm{Q}$ must be calculated, which is obtained as:

$$
\varepsilon_{\mathrm{P}}^{\lambda}=\frac{\mathrm{A}_{\mathrm{P}}^{\lambda}}{\mathrm{l} \mathrm{c}_{\mathrm{P}}} \quad \varepsilon_{\mathrm{Q}}^{\lambda}=\frac{\mathrm{A}_{\mathrm{Q}}^{\lambda}}{\mathrm{lc} \mathrm{c}_{\mathrm{Q}}}
$$

The absorption spectrum in UV-Visible regions is influenced by three factors: concentration, temperature, and $\mathrm{pH}$

\section{B. Qualitative Analysis}

When a matter is irradiated with energy of radiation, a number process occurs such as reflection, scattering, absorbance, fluorescence, phosphorescence and photochemical reaction. In UV-Visible spectra, only absorption process is emphasized; i.e., with the absorption of light, the energy of molecules increases. The potential energy of a molecule is given by:

and

$$
\mathrm{E}_{\text {total }}=\mathrm{E}_{\text {electronic }}+\mathrm{E}_{\text {vibrational }}+\mathrm{E}_{\text {rotational }}
$$

$$
\mathrm{E}_{\text {electronic }}>\mathrm{E}_{\text {vibrational }}>\mathrm{E}_{\text {rotational }}
$$

The features of UV-Visible absorption are: a) all molecules are in vibrational ground state at room temperature, b) transition among different energy states is induced by electromagnetic radiation in UV-Visible region, c) each electronic states is accompanied with vibrational and rotational levels, d) Boltzmann distribution will be applied, in order to determine the relative populations of various energy states, as different molecules absorbs different wavelength of radiation, the frequency of absorbed light dependent on the structure of the molecule. Usually, coarse structure in the spectra depicts vibrational changes, while fine structure in spectra is due to rotational changes [6].

In UV-Visible spectroscopy, bands are generally broad. There are three types of electronic transitions: $\sigma$ bonding, $\pi$ bonding and $\mathrm{n}$ bonding. In addition, two types of antibonding orbitals may be involved transition $\sigma^{*}$ and $\pi^{*}$ orbital. The $\sigma$ orbitals are the occupied orbital with lowest energy, while $\sigma^{*}$ anti-bonding orbital are unoccupied higher energy levels. $\pi$ are occupied orbitals with highest energy, while $\pi^{*}$ anti-bonding orbtials are unoccupied lower energy compared to $\sigma^{*}$. Atomic orbitals with $\mathrm{n}$ orbital are with nonbonding electrons with energy higher than $\sigma$ and $\pi$. A $\sigma \rightarrow$ $\sigma^{*}$ in which a bonding of s electron is excited to an antibonding orbital, while a transition of one electron of a lone pair to an anti-bonding $\pi$ orbital is referred to as $\pi \rightarrow \pi^{*}$. Thus, the following electronic transition can occur by the absorption of UV-Visible light: $\sigma \rightarrow \sigma^{*}, \mathrm{n} \rightarrow \sigma^{*}, \mathrm{n} \rightarrow \pi^{*}$ and $\pi \rightarrow \pi^{*}$ [7]. Table I shows the transition type of organic, inorganic and metal-ligand complex. It is to note that for organic (saturated or unsaturated) transition involves electrons: $\pi, \sigma$ and $n$; the inorganic compounds involves electron: $\mathrm{d}$ and $\mathrm{f}$ orbitals. The metal ligand complex is as a result of charge transfer. Table II shows the transition range common to all molecules resulting in simple bonds.

\section{EXPERIMENTAL METHOD}

There are three types of instruments used for the analysis of a compound in UV-visible region. They are: a) single beam spectrometer, b) double beam spectrometer, and c) simultaneous spectrometer. Fig. 2 below shows the double beam spectrometer.

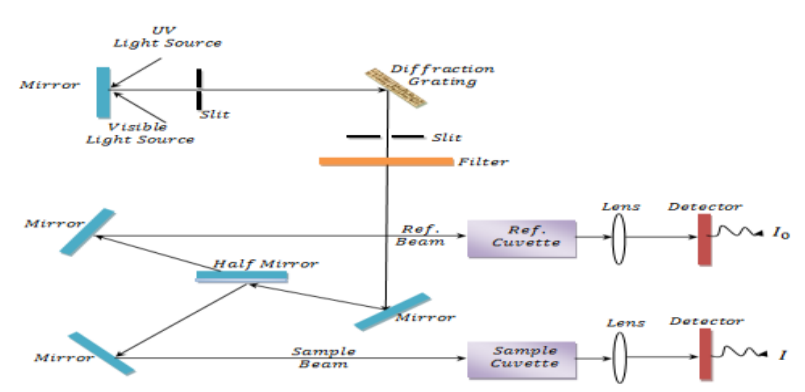

Fig. 2. Double beam spectrometer for UV-Visible Region

UV-Visible spectroscopic data provides both qualitative and quantitative information about a compound. A calibration curve is prepared by using at least five different concentrations of the compound. A graph of absorbance versus wavelength and highest absorbance versus concentration is plotted. Fig. 3 shows the UV-Visible spectra and calibration curve for of different concentration. 


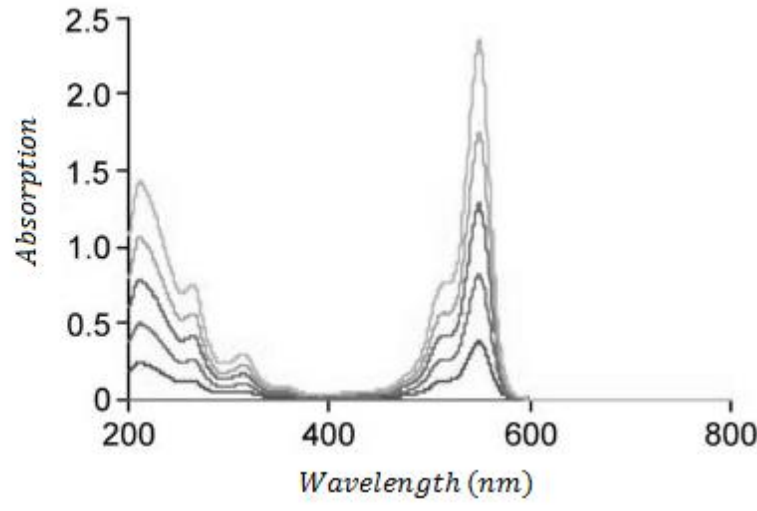

Fig. 3(a). Absorbance versus wave length

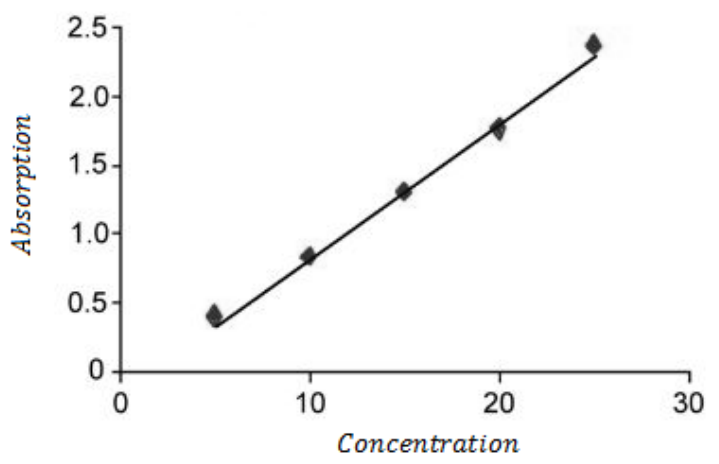

Fig. 3(b). Absorbance versus concentration

\section{EMPIRICAL DATA IN UV-VISIBLE REGION}

TABLE I: DATA ON TRANSITION TYPE OF DIFFERENT COMPOUNDS

\begin{tabular}{llc}
\hline \hline Compound & Nature & Transition \\
\hline & Saturated & $\sigma \rightarrow \sigma^{*}$ \\
Organic & & $n \rightarrow \sigma^{*}$ \\
& Unsaturated & $\mathrm{n} \rightarrow \pi^{*}$ \\
& Electron transfer takes places from a donor to an \\
Metal - Ligand & acceptor. Either metal or ligand may be donor or \\
Complexes & acceptor & \\
Inorganic & Transition among d-orbitals are splitted by the presence \\
& of ligand field. Air absorbs wavelength 200 $\mathrm{nm}$.
\end{tabular}

TABLE II: DATA 9N TRANSITION WITH WAVELENGTH AND WAVE NUMBER FOR MOLECULES

\begin{tabular}{ccc}
\hline \hline Transition & Wavelength, $n m$ & $\begin{array}{c}\text { Wave number, } \\
\left(10^{4} \mathrm{~cm}^{-1}\right)\end{array}$ \\
\hline$\sigma \rightarrow \sigma^{*}$ & $20-200$ & $9.80-5.80$ \\
$\mathrm{n} \rightarrow \sigma^{*}$ & $150-260$ & $5.05-3.50$ \\
$\pi \rightarrow \pi^{*}$ & $180-240$ & $5.10-4.12$ \\
$($ Isolated) & $220-300$ & $4.20-3.37$ \\
$\pi \rightarrow \pi^{*}$ & $220-800$ & \\
$(1,3$ Dienes) & $250-800$ & $4.25-1.33$ \\
$\pi \rightarrow \pi^{*}$ & & $3.65-1.25$ \\
$($ conjugated) &
\end{tabular}

The molar absorption coefficient $\varepsilon$ at a particular wavelength corresponds maximum absorption. The value of $\varepsilon$ depends on the solvent used in the analysis. Table III shows the value of molar absorption coefficient $\varepsilon$ of different compound.
TABLE III: DATA ON MOLAR ABSORPTIVITY ( E ) FOR ORGANIC COMPOUNDS

\begin{tabular}{cc}
\hline \hline Compound & $\varepsilon\left(10^{4} \mathrm{~L} \mathrm{~mol}^{-1} \mathrm{~cm}^{-1}\right)$ \\
\hline Acridine & 1.20 \\
Acridine orange & 3.00 \\
Antracene & 1.00 \\
Benzene & 0.02 \\
Bianthryl & 2.40 \\
Biphenyl & 1.60 \\
Carbazole & 4.20 \\
Eosin Y & 9.00 \\
Fluorene & 9.00 \\
Indole & 5.50 \\
Phenol & 2.00 \\
Perylene & 3.40 \\
Rhodamine B & 1.05 \\
\hline \hline
\end{tabular}

\section{DISCUSSION}

Spectroscopy is an important qualitative and quantitative analysis of atoms and molecules. With the advent of modern technologies, the spectroscopic techniques have developed to a larger extent. There exist many spectroscopic techniques, one of the basic spectroscopic technique is UVVisible spectroscopy, which is widely used in chemistry and life sciences for the analysis of different organic and inorganic compounds. The UV-Visible spectroscopic technique is importantly used for the determination of concentration of molecules in a solution, concentration of cells in microbiology and in identification the functional/chemical groups presents in a solution, identification of conjugated organic compounds, metal ions in transition states and kinetics of enzymes.

The energy of radiation is dependent on frequency, numerically $300 \mathrm{~kJ} \mathrm{~mol}^{-1}$ for blue light and $170 \mathrm{~kJ} \mathrm{~mol}^{-1}$ for red light in the visible region. The transition of electrons among different levels is due to absorption and emission of incident radiation, these electronic transitions are predominant in UV and visible region of electromagnetic spectrum. For this reason, the spectrum between $200 \mathrm{~nm}$ to $800 \mathrm{~nm}$ is commonly used to encompass all the electronic transitions. The UV-Visible regions is based on Beer's Lambert Law given by equation (1). The energies of orbitals involved in electronic transitions are discrete, and the absorption spectrum should have sharp peaks. Instead, the absorption spectrum is observed to have peak broadening, this is due to presence of vibrational energies.

Due to the presence of particular function group, many organic molecules absorbs UV-visible radiation, these function group are termed as chromophores. There are different ways of determining the organic compounds: qualitatively by identifying electronic transitions involved, and quantitatively by measuring absorbance and concentration of the solution. However, when the concentration and absorbance of a solution is known, one may determine the value of molar absorption coefficient of the solution. The molar absorption coefficient is an intrinsic property of species; the molar absorptivity of different organic compounds is shown in Table III. Thus, with the determination of molar absorption coefficient, one can validate the organic compound present in the solution. However, it is to note that the molar absorption coefficient depends on concentration, temperature and $\mathrm{pH}$, as well as 
partially depends on the characteristics of the instrument used.

In past, there have been a tremendous deviation in understanding qualitative and quantitative analysis of compounds using infra-red (IR) spectroscopy. IR spectroscopy give a detailed insights of the functional group present in the compound. One can easily distinguish groups such as carbonyls, hydroxyl, carboxyl, double and triple bonds. On the other hand, UV-visible spectrometric technique serves as a quick and easy tool in quality analysis, with some limitations such as chemical deviations and scattered radiation: the former is due to chemical phenomena involving association, dissociation and interaction of molecules, and the later is due to reflection and scattering of radiation by lens surface, gratings, filters and windows. Thus, UV-visible spectra generally serve as a tool for validating of organic compounds in quickly and easily.

\section{REFERENCES}

[1] Helmut Gunzler \& Alex Williams, Ultraviolet and visible spectroscopy. In Handbook of Analytical Techniques: Wiley-VCH Weinheim, Germany, 2001.

[2] Borys Jagielski, "Elements of the wave particle duality of light," Master thesis, University of Oslo, Oslo, 2009

[3] Corey N. Stedwell and Nicolas C. Polfer, "Laser photo dissociation and spectroscopy of mass separated bimolecular ions," Lecture notes in chemistry, No. 83, 2013.

[4] Frederic R. Stauffer and Hajime Sakai, "Derivative Spectroscopy," Appl. Opt., Vol. 7, No. 1, pp. 61-65, 1968.

[5] Tony Owen, "Fundamental of modern UV visible spectroscopy," Hewlett Packard company, Germany, pp. 79-84, 1996.

[6] Klaus von Haeften, A. R. B. De Castro, M. Joppien, L Moussavizadeh, R. von Pietrowski and Thomas Moller, "The role of electronic, vibrational and rotational energy transfer," Vol. 78, No. 23, pp. 4371, 1997.

[7] Subodh Kumar, "Spectroscopy of organic compounds," New Age International Pvt. Ltd., pp. 8-10, 2006. 